1 Hacettepe Journal of Mathematics and Statistics

h Volume 46 (5) (2017), 817-828

\title{
Response of a 3D elastic half-space to a distributed moving load
}

\author{
Nihal Ege*†, Onur Şahin ${ }^{\ddagger}$ and Barış Erbaş§
}

\begin{abstract}
The dynamic effect of an out of plane distributed moving load on the surface of an elastic half-space is considered. The problem is formulated in terms of a hyperbolic-elliptic asymptotic model for a moving load where the trajectory and the distribution of the load are taken to be orthogonal. Steady-state equations are written in terms of a moving coordinate system. The near-resonant solutions are, then, obtained for sub and super-Rayleigh cases taking into account the causality principle. Numerical results of displacement components are presented for various values of the distribution parameter.
\end{abstract}

Keywords: Moving load, Asymptotic model, Rayleigh wave, Causality. 2000 AMS Classification: 74J15, 74G10, 74B05.

Received : 01.09.2016 Accepted : 21.12.2016 Doi : 10.15672 /HJMS.2017.434

\section{Introduction}

Surface wave phenomena has been an active research area since the pioneering work of Rayleigh in 1887. There are numerous publications in the literature analyzing the response of elastic structures under the influence of both stationary and moving loads [1]-[4]. However a great number of these studies consider only two dimensional problems and there exists only a small number of papers analyzing 3D problems. Furthermore, the applied forces are taken as point loads which do not reflect real life problems encountered in modern engineering applications such as operation of high speed trains ([5], [6]), underground railway tunnels ([7]), bridge constructions ([8]), etc.

\footnotetext{
*Anadolu University, Department of Mathematics, Yunus Emre Campus, 26470, Eskişehir Email: nsahin@anadolu.edu.tr

$\dagger_{\text {Corresponding Author. }}$

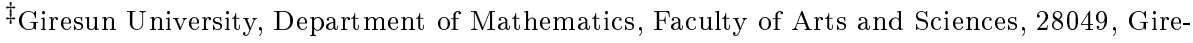
sun, Email: onur.sahin@giresun.edu.tr

$\S$ Anadolu University, Department of Mathematics, Yunus Emre Campus, 26470, Eskişehir , Email: berbas@anadolu.edu.tr
} 
The main object of this paper is to extract the contribution of the Rayleigh wave resulting from a moving load of Gaussian-type profile distributed orthogonally to the trajectory of the load. Recently, a similar problem has been analyzed by the authors where the distribution and motion of the moving load are taken in the same direction (cf. [9]). The direction of the distribution considered here, contrary to the previously examined case, gives rise to significant difficulties in obtaining the solution in particular cases. Since the focus will be on the surface displacement, an approximate model developed by [10] and later implemented to 3D in [11]-[13] will be employed in order to analyse the surface wave contribution ignoring the effects of bulk and shear waves. The advantage of the adapted model is that it reduces the governing equations to a mixed type surface equation depending on the load speed and two 2D equations in the interior of the half-space. Moreover the model enables one to obtain explicit expressions for stress and displacement components.

The paper is organised as follows: In Section 2 the statement of the problem is formulated in terms of the adapted asymptotic model. Introducing a moving coordinate system, steady-state equations are obtained which are then cast into sub and superRayleigh cases determined by the small parameter defining the proximity of the load speed to the Rayleigh wave speed. In Section 3, the surface solutions are obtained and only in the sub-Rayleigh case the interior solution is restored in terms of elementary functions. However, due to the nature of the load, such an extension does not result in an immediate explicit solution in the super-Rayleigh case. This demands expressing the interior solution in terms of an integral form. Finally, Section 4 represents the illustrations of the displacements depending on the variation of the load distribution, as well as depth of the half-space.

\section{Statement of the problem}

Consider a linearly elastic isotropic half-space, occupying the domain $-\infty<x_{1}, x_{2}<\infty, 0 \leqslant x_{3}<\infty$, subject to a vertical load of magnitude $P$ distributed along the $O x_{2}$ axis and moving along the $O x_{1}$ along the surface $x_{3}=0$ of the half-space at a constant speed $c$, see Figure 1 .

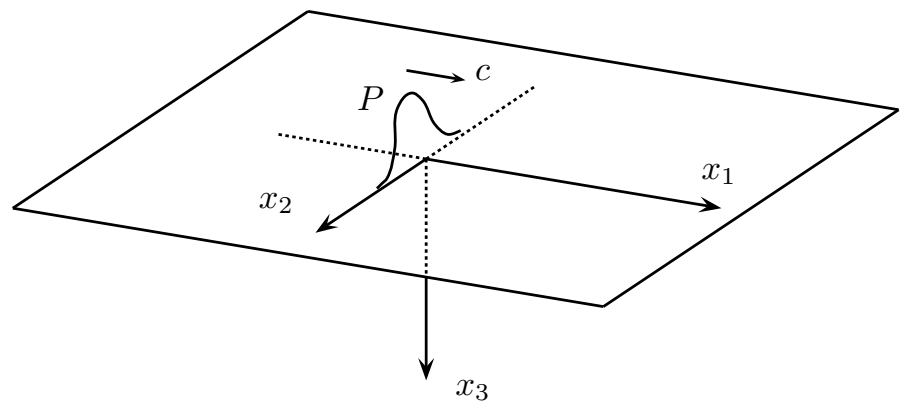

Figure 1. Distributed load moving along $O x_{1}$ axis.

The equations of motion in linear elasticity are given in their conventional form (see [14]),

$$
(\lambda+\mu) \operatorname{grad} \operatorname{div} \mathbf{u}+\mu \Delta \mathbf{u}=\rho \frac{\partial^{2} \mathbf{u}}{\partial t^{2}},
$$


where $\lambda$ and $\mu$ are Lamé constants, $\rho$ is the volume density, $\mathbf{u}$ is the displacement vector and $\Delta$ is a 3 -dimensional Laplace operator.

In the framework of the elliptic-hyperbolic asymptotic model, derived in [15] and [16], the elliptic equations for the interior can be presented as

$$
\frac{\partial^{2} \phi}{\partial x_{3}^{2}}+k_{1}^{2} \Delta_{2} \varphi=0, \quad \frac{\partial^{2} \psi_{i}}{\partial x_{3}^{2}}+k_{2}^{2} \Delta_{2} \psi_{i}=0, \quad i=1,2
$$

where

$$
\Delta_{2}=\frac{\partial^{2}}{\partial x_{1}^{2}}+\frac{\partial^{2}}{\partial x_{2}^{2}}, \quad k_{i}^{2}=1-\frac{c_{R}^{2}}{c_{i}^{2}},
$$

and $c_{1}, c_{2}$, and $c_{R}$ are the longitudinal, shear and Rayleigh wave speeds respectively.

The boundary equation along the surface $x_{3}=0$ is governed by a hyperbolic equation

$$
\Delta_{2} \varphi-\frac{1}{c_{R}^{2}} \frac{\partial^{2} \varphi}{\partial t^{2}}=A P \delta\left(x_{1}-c t\right) \frac{a}{\pi\left(x_{2}^{2}+a^{2}\right)},
$$

where $\delta(x)$ is the Dirac delta function and $A$ is a material constant defined in [11]. The longitudinal and transverse potentials $\varphi$ and $\psi_{i}$ are connected by a differential relation on the surface $x_{3}=0$ given by (cf. [11])

$$
\frac{\partial \varphi}{\partial x_{i}}=\frac{2}{1+k_{2}^{2}} \frac{\partial \psi_{i}}{\partial x_{3}}, \quad i=1,2
$$

On using the well-known Helmholtz decomposition theorem, the components of the displacement vector may be rewritten in terms of the wave potentials as

$$
u_{1}=\frac{\partial \varphi}{\partial x_{1}}-\frac{\partial \psi_{1}}{\partial x_{3}}, \quad u_{2}=\frac{\partial \varphi}{\partial x_{2}}-\frac{\partial \psi_{2}}{\partial x_{3}}, \quad u_{3}=\frac{\partial \varphi}{\partial x_{3}}+\frac{\partial \psi_{1}}{\partial x_{1}}+\frac{\partial \psi_{2}}{\partial x_{2}} .
$$

Let us now employ a moving coordinate $\lambda=x_{1}-c t$ which restricts the problem to a steady-state regime. Introducing a small dimensionless parameter, $\varepsilon$, defined by

$$
\varepsilon=\sqrt{\left|1-\frac{c^{2}}{c_{R}^{2}}\right|}
$$

the boundary equation (2.3) reduces to an elliptic equation

$$
\frac{\partial^{2} \varphi}{\partial x_{2}^{2}}+\varepsilon^{2} \frac{\partial^{2} \varphi}{\partial \lambda^{2}}=A P \delta(\lambda) \frac{a}{\pi\left(x_{2}^{2}+a^{2}\right)},
$$

corresponding to a sub-Rayleigh regime $\left(c<c_{R}\right)$ and to a hyperbolic equation

$$
\frac{\partial^{2} \varphi}{\partial x_{2}^{2}}-\varepsilon^{2} \frac{\partial^{2} \varphi}{\partial \lambda^{2}}=A P \delta(\lambda) \frac{a}{\pi\left(x_{2}^{2}+a^{2}\right)},
$$

corresponding to a super-Rayleigh regime $\left(c>c_{R}\right)$.

It is worth noting that the adapted model for a $3 \mathrm{D}$ elastic half-space, presented in [11] and [16] , is valid provided that $\varepsilon \ll 1$, i.e., when the load speed is close to the Rayleigh wave speed. Due to the type of the applied load and its direction of movement, it is not unreasonable to expect that the main displacements will occur in $x_{1}$ and $x_{3}$ directions.

The presence of a small physical parameter together with the above reasoning, thus, motivates the scaling

$$
\eta_{1}=\frac{\lambda}{\varepsilon}, \quad \eta_{2}=x_{2}, \quad \eta_{3}=\frac{x_{3}}{\varepsilon} .
$$

Utilizing (2.9), equations (2.2), (2.4), (2.7) and (2.8) become

$$
\frac{\partial^{2} \varphi}{\partial \eta_{3}^{2}}+k_{1}^{2} \frac{\partial^{2} \varphi}{\partial \eta_{1}^{2}}+\varepsilon^{2} k_{1}^{2} \frac{\partial^{2} \varphi}{\partial \eta_{2}^{2}}=0, \quad \frac{\partial^{2} \psi_{i}}{\partial \eta_{3}^{2}}+k_{2}^{2} \frac{\partial^{2} \psi_{i}}{\partial \eta_{1}^{2}}+\varepsilon^{2} k_{2}^{2} \frac{\partial^{2} \psi_{i}}{\partial \eta_{2}^{2}}=0
$$




$$
\begin{aligned}
& \frac{\partial \varphi}{\partial \eta_{1}}=\frac{2}{1+k_{2}^{2}} \frac{\partial \psi_{1}}{\partial \eta_{3}}, \quad \frac{\partial \varphi}{\partial \eta_{2}}=\frac{2}{\varepsilon\left(1+k_{2}^{2}\right)} \frac{\partial \psi_{2}}{\partial \eta_{3}} \\
& \frac{\partial^{2} \varphi}{\partial \eta_{2}^{2}}+\frac{\partial^{2} \varphi}{\partial \eta_{1}^{2}}=\frac{A P}{\varepsilon} \delta\left(\eta_{1}\right) \frac{a}{\pi\left(\eta_{2}^{2}+a^{2}\right)}
\end{aligned}
$$

and

$$
\frac{\partial^{2} \varphi}{\partial \eta_{2}^{2}}-\frac{\partial^{2} \varphi}{\partial \eta_{1}^{2}}=\frac{A P}{\varepsilon} \delta\left(\eta_{1}\right) \frac{a}{\pi\left(\eta_{2}^{2}+a^{2}\right)},
$$

respectively.

\section{Solution of the problem}

In this section the steady-state solutions of equations (2.10)-(2.13) is derived by using the approximate hyperbolic-elliptic model. We first neglect $\mathrm{O}\left(\varepsilon^{2}\right)$ terms in the elliptic equations (2.10), resulting in

$$
\frac{\partial^{2} \varphi}{\partial \eta_{3}^{2}}+k_{1}^{2} \frac{\partial^{2} \varphi}{\partial \eta_{1}^{2}}=0, \quad \frac{\partial^{2} \psi_{i}}{\partial \eta_{3}^{2}}+k_{2}^{2} \frac{\partial^{2} \psi_{i}}{\partial \eta_{1}^{2}}=0 .
$$

Thus, the problem is reduced to a pair of $2 \mathrm{D}$ boundary value problems given by (3.1) together with the boundary conditions (2.12) and (2.13), corresponding to sub and superRayleigh cases, respectively, whose solutions will be the main consideration of the following discussion.

3.1. Sub-Rayleigh case. We first obtain the solution of the boundary equation (2.12). To this end, we will employ the fundamental solution of the Laplace operator, see [17], i.e.,

$$
\mathscr{F}\left(\eta_{1}, \eta_{2}\right)=\frac{1}{4 \pi} \ln \left(\eta_{1}^{2}+\eta_{2}^{2}\right),
$$

and convolute it with the right hand side of (2.12) giving the solution of the longitudinal potential on the surface written as

$$
\begin{aligned}
\varphi\left(\eta_{1}, \eta_{2}\right) & =\mathscr{F}\left(\eta_{1}, \eta_{2}\right) * \frac{A P}{\varepsilon} \delta\left(\eta_{1}\right) \frac{a}{\pi\left(\eta_{2}^{2}+a^{2}\right)} \\
& =\frac{A P a}{4 \pi^{2} \varepsilon} \int_{-\infty}^{\infty} \int_{-\infty}^{\infty} \frac{\ln \left(\xi_{1}^{2}+\xi_{2}^{2}\right)}{\left(\eta_{2}-\xi_{2}\right)^{2}+a^{2}} \delta\left(\eta_{1}-\xi_{1}\right) d \xi_{1} d \xi_{2} \\
& =\frac{A P a}{4 \pi^{2} \varepsilon} \int_{-\infty}^{\infty} \frac{\ln \left(\eta_{1}^{2}+\xi_{2}^{2}\right)}{\left(\eta_{2}-\xi_{2}\right)^{2}+a^{2}} d \xi_{2} .
\end{aligned}
$$

The integral in the last line of equation (3.3) may straightforwardly be calculated using the residue calculus, resulting in

$$
\varphi\left(\eta_{1}, \eta_{2}\right)=\frac{A P}{4 \pi \varepsilon} \ln \left(\eta_{2}^{2}+\left(a+\left|\eta_{1}\right|\right)^{2}\right), \quad \eta_{3}=0 .
$$

The solution of the elliptic equation (3.1) over the interior $\eta_{3}>0$ can be obtained through the use of Poisson's formula (see [18]), with the boundary value (3.4), which gives

$$
\varphi\left(\eta_{1}, \eta_{2}, \eta_{3}\right)=\frac{A P}{4 \pi^{2} \varepsilon} k_{1} \eta_{3} \int_{-\infty}^{\infty} \frac{\ln \left((a+|\xi|)^{2}+\eta_{2}^{2}\right)}{\left(\xi-\eta_{1}\right)^{2}+k_{1}^{2} \eta_{3}^{2}} d \xi,
$$


once again, applying a simple contour integration we find that

$$
\begin{aligned}
\varphi\left(\eta_{1}, \eta_{2}, \eta_{3}\right)=\frac{A P}{4 \pi \varepsilon}[ & \ln \left(\left(a+\eta_{1}\right)^{2}+\left(k_{1} \eta_{3}+\left|\eta_{2}\right|\right)^{2}\right)+ \\
& \left.+\ln \left(\left(a-\eta_{1}\right)^{2}+\left(k_{1} \eta_{3}+\left|\eta_{2}\right|\right)^{2}\right)\right] .
\end{aligned}
$$

The transverse potentials $\psi_{1}$ and $\psi_{2}$ can be determined with the help of differential relations (2.11). Differentiation of (3.5) gives

$$
\begin{aligned}
\frac{\partial \varphi\left(\eta_{1}, \eta_{2}, \eta_{3}\right)}{\partial \eta_{1}}=\frac{A P}{2 \pi \varepsilon}[ & {\left[\frac{a+\eta_{1}}{\left(a+\eta_{1}\right)^{2}+\left(k_{1} \eta_{3}+\left|\eta_{2}\right|\right)^{2}}\right.} \\
& \left.-\frac{a-\eta_{1}}{\left(a-\eta_{1}\right)^{2}+\left(k_{1} \eta_{3}+\left|\eta_{2}\right|\right)^{2}}\right] .
\end{aligned}
$$

Taking into consideration $\left(3.1_{2}\right)$ and $\left(2.11_{1}\right)$ and using the harmonicity of the Lamé potentials (see [4]; also [19]) we obtain

$$
\begin{aligned}
\frac{\partial \psi_{1}\left(\eta_{1}, \eta_{2}, \eta_{3}\right)}{\partial \eta_{3}}=\frac{A P\left(1+k_{2}^{2}\right)}{4 \pi \varepsilon}[ & \frac{a+\eta_{1}}{\left(a+\eta_{1}\right)^{2}+\left(k_{2} \eta_{3}+\left|\eta_{2}\right|\right)^{2}} \\
& \left.-\frac{a-\eta_{1}}{\left(a-\eta_{1}\right)^{2}+\left(k_{2} \eta_{3}+\left|\eta_{2}\right|\right)^{2}}\right],
\end{aligned}
$$

hence,

$$
\begin{aligned}
\psi_{1}\left(\eta_{1}, \eta_{2}, \eta_{3}\right)=\frac{A P\left(1+k_{2}^{2}\right)}{4 \pi \varepsilon k_{2}}[ & \arctan \left(\frac{k_{2} \eta_{3}+\left|\eta_{2}\right|}{a+\eta_{1}}\right) \\
& \left.-\arctan \left(\frac{k_{2} \eta_{3}+\left|\eta_{2}\right|}{a-\eta_{1}}\right)\right] .
\end{aligned}
$$

In a similar manner, considering $\left(3.1_{2}\right)$ and $\left(2.11_{2}\right)$, we get

$$
\begin{aligned}
\frac{\partial \psi_{2}\left(\eta_{1}, \eta_{2}, \eta_{3}\right)}{\partial \eta_{3}}=\frac{A P\left(1+k_{2}^{2}\right) \operatorname{sgn}\left(\eta_{2}\right)}{4 \pi}[ & \frac{k_{2} \eta_{3}+\left|\eta_{2}\right|}{\left(a+\eta_{1}\right)^{2}+\left(k_{2} \eta_{3}+\left|\eta_{2}\right|\right)^{2}} \\
& \left.+\frac{k_{2} \eta_{3}+\left|\eta_{2}\right|}{\left(a-\eta_{1}\right)^{2}+\left(k_{2} \eta_{3}+\left|\eta_{2}\right|\right)^{2}}\right],
\end{aligned}
$$

from which it follows that

$$
\begin{aligned}
\psi_{2}\left(\eta_{1}, \eta_{2}, \eta_{3}\right)=\frac{A P\left(1+k_{2}^{2}\right) \operatorname{sgn}\left(\eta_{2}\right)}{8 \pi k_{2}} & {\left[\ln \left(\left(a+\eta_{1}\right)^{2}+\left(k_{2} \eta_{3}+\left|\eta_{2}\right|\right)^{2}\right)+\right.} \\
+ & \left.\ln \left(\left(a-\eta_{1}\right)^{2}+\left(k_{2} \eta_{3}+\left|\eta_{2}\right|\right)^{2}\right)\right] .
\end{aligned}
$$

The components of the displacement vector $\mathbf{u}$ are rewritten in terms of scaled variables (2.9) as

$$
\begin{array}{r}
u_{1}=\frac{1}{\varepsilon}\left(\frac{\partial \varphi}{\partial \eta_{1}}-\frac{\partial \psi_{1}}{\partial \eta_{3}}\right), \quad u_{2}=\frac{\partial \varphi}{\partial \eta_{2}}-\frac{1}{\varepsilon} \frac{\partial \psi_{2}}{\partial \eta_{3}} \\
u_{3}=\frac{1}{\varepsilon}\left(\frac{\partial \varphi}{\partial \eta_{3}}+\frac{\partial \psi_{1}}{\partial \eta_{1}}\right)+\frac{1}{a} \frac{\partial \psi_{2}}{\partial \eta_{2}}
\end{array}
$$

Substitution of equations (3.5), (3.8), and (3.10) into the displacement components gives

$$
\begin{aligned}
u_{1} & =\frac{1}{\varepsilon^{2}} \frac{A P}{2 \pi}\left[\frac{a+\eta_{1}}{\left(a+\eta_{1}\right)^{2}+\left(k_{1} \eta_{3}+\left|\eta_{2}\right|\right)^{2}}-\frac{a-\eta_{1}}{\left(a-\eta_{1}\right)^{2}+\left(k_{1} \eta_{3}+\left|\eta_{2}\right|\right)^{2}}-\right. \\
& \left.-\frac{1+k_{2}^{2}}{2}\left(\frac{a+\eta_{1}}{\left(a+\eta_{1}\right)^{2}+\left(k_{2} \eta_{3}+\left|\eta_{2}\right|\right)^{2}}-\frac{a-\eta_{1}}{\left(a-\eta_{1}\right)^{2}+\left(k_{2} \eta_{3}+\left|\eta_{2}\right|\right)^{2}}\right)\right],
\end{aligned}
$$




$$
\begin{aligned}
u_{2} & =\frac{1}{\varepsilon} \frac{A P \operatorname{sgn}\left(\eta_{2}\right)}{2 \pi}\left[\frac{k_{1} \eta_{3}+\left|\eta_{2}\right|}{\left(a+\eta_{1}\right)^{2}+\left(k_{1} \eta_{3}+\left|\eta_{2}\right|\right)^{2}}+\frac{k_{1} \eta_{3}+\left|\eta_{2}\right|}{\left(a-\eta_{1}\right)^{2}+\left(k_{1} \eta_{3}+\left|\eta_{2}\right|\right)^{2}}-\right. \\
& \left.-\frac{1+k_{2}^{2}}{2}\left(\frac{k_{2} \eta_{3}+\left|\eta_{2}\right|}{\left(a+\eta_{1}\right)^{2}+\left(k_{2} \eta_{3}+\left|\eta_{2}\right|\right)^{2}}+\frac{k_{2} \eta_{3}+\left|\eta_{2}\right|}{\left(a-\eta_{1}\right)^{2}+\left(k_{2} \eta_{3}+\left|\eta_{2}\right|\right)^{2}}\right)\right]
\end{aligned}
$$

and

$$
\begin{aligned}
u_{3} & =\frac{A P\left(1+k_{2}^{2}\right)}{4 \pi k_{2}}\left[\frac{k_{2} \eta_{3}+\left|\eta_{2}\right|}{\left(a+\eta_{1}\right)^{2}+\left(k_{2} \eta_{3}+\left|\eta_{2}\right|\right)^{2}}+\frac{k_{2} \eta_{3}+\left|\eta_{2}\right|}{\left(a-\eta_{1}\right)^{2}+\left(k_{2} \eta_{3}+\left|\eta_{2}\right|\right)^{2}}\right] \\
& +\frac{1}{\varepsilon^{2}} \frac{A P}{2 \pi}\left[k_{1}\left(\frac{\left.k_{1} \eta_{3}+\left|\eta_{2}\right|\right)}{\left(a+\eta_{1}\right)^{2}+\left(k_{1} \eta_{3}+\left|\eta_{2}\right|\right)^{2}}+\frac{\left.k_{1} \eta_{3}+\left|\eta_{2}\right|\right)}{\left(a-\eta_{1}\right)^{2}+\left(k_{1} \eta_{3}+\left|\eta_{2}\right|\right)^{2}}\right)\right. \\
& \left.-\frac{1+k_{2}^{2}}{2 k_{2}}\left(\frac{k_{2} \eta_{3}+\left|\eta_{2}\right|}{\left(a+\eta_{1}\right)^{2}+\left(k_{2} \eta_{3}+\left|\eta_{2}\right|\right)^{2}}+\frac{k_{2} \eta_{3}+\left|\eta_{2}\right|}{\left(a-\eta_{1}\right)^{2}+\left(k_{2} \eta_{3}+\left|\eta_{2}\right|\right)^{2}}\right)\right] .
\end{aligned}
$$

Equations (3.12)-(3.14) express the asymptotic solutions of the displacement components in the sub-Rayleigh case. Keeping $\mathrm{O}\left(\varepsilon^{2}\right)$ terms in elliptic equations (2.10), the exact solution of the 3D boundary value problem is again obtained with the help of the Poisson's formula in an integral form as

$$
\varphi\left(\eta_{1}, \eta_{2}, \eta_{3}\right)=\frac{A P \eta_{3}}{8 \pi^{2} \varepsilon} \int_{-\infty}^{\infty} \int_{-\infty}^{\infty} \frac{\ln \left(\xi_{2}^{2}+\left(\frac{a}{\varepsilon k_{1}}+\left|\frac{\xi_{1}}{\varepsilon}\right|\right)^{2}\right)}{\left[\left(\xi_{1}-\frac{\eta_{1}}{k_{1}}\right)^{2}+\left(\xi_{2}-\frac{\eta_{2}}{\varepsilon k_{1}}\right)^{2}+\eta_{3}^{2}\right]^{3 / 2}} d \xi_{1} d \xi_{2}
$$

3.2. Super-Rayleigh case. We now proceed to the super-Rayleigh case. Following a similar approach as in the foregoing discussion the solution of the boundary equation (2.13) may be obtained using the fundamental solution of the wave operator, see [17]. It is critical to note, at this point, that since the load speed is greater than the Rayleigh wave speed we should not expect any disturbance on the surface in front of the load, a fact dictating us to employ the causality principle (for more details, see [9]). The fundamental solution of the 1D wave operator, therefore, takes the form

$$
\mathscr{E}\left(\eta_{1}, \eta_{2}\right)=\frac{1}{2}\left[H\left(\eta_{2}-\eta_{1}\right)-H\left(\eta_{2}+\eta_{1}\right)\right] H\left(-\eta_{1}\right) .
$$

Thus, the longitudinal wave potential on the surface $\eta_{3}=0$ can be obtained through a convolution as

$$
\begin{aligned}
\varphi\left(\eta_{1}, \eta_{2}\right) & =\mathscr{E}\left(\eta_{1}, \eta_{2}\right) * \frac{A P}{\varepsilon} \delta\left(\eta_{1}\right) \frac{1}{\pi\left(\eta_{2}^{2}+1\right)} \\
& =\frac{A P}{2 \pi \varepsilon} \int_{-\infty}^{\infty} \int_{-\infty}^{0} \frac{H\left(\xi_{2}-\xi_{1}\right)-H\left(\xi_{2}+\xi_{1}\right)}{\left(\eta_{2}-\xi_{2}\right)^{2}+1} \delta\left(\eta_{1}-\xi_{1}\right) d \xi_{1} d \xi_{2} \\
& =\frac{A P}{2 \pi \varepsilon}\left[\arctan \left(\frac{\eta_{2}-\eta_{1}}{a}\right)-\arctan \left(\frac{\eta_{2}+\eta_{1}}{a}\right)\right] H\left(-\eta_{1}\right) .
\end{aligned}
$$

The solution over the interior follows from the Poisson's formula and is given as

$$
\varphi\left(\eta_{1}, \eta_{2}, \eta_{3}\right)=\frac{A P}{2 \pi^{2} \varepsilon} k_{1} \eta_{3} \int_{-\infty}^{0} \frac{\arctan \left(\frac{\eta_{2}-\xi}{a}\right)-\arctan \left(\frac{\eta_{2}+\xi}{a}\right)}{\left(\xi-\eta_{1}\right)^{2}+k_{1}^{2} \eta_{3}^{2}} d \xi
$$

As it is quite difficult, if not impossible, to calculate the integral in equation (3.18) analytically, we will only concentrate on the surface displacement since it is possible to express them through the relations (2.11) given analytical formulae. The longitudinal 
and transverse displacements along the surface $x_{3}=0$ can therefore be written from equations (2.5) and (2.11) as

$$
u_{1}=\frac{c_{R}^{2}}{2 \varepsilon c_{2}^{2}} \frac{\partial \varphi}{\partial \eta_{1}}, \quad u_{2}=\frac{c_{R}^{2}}{2 c_{2}^{2}} \frac{\partial \varphi}{\partial \eta_{2}} .
$$

Hence, the tangential displacements along the plane $x_{3}=0$ are expressed through the formulae

$$
\begin{aligned}
& u_{1}=-\frac{A P c_{R}^{2} a}{4 \pi \varepsilon^{2} c_{2}^{2}} {\left[\left(\arctan \left(\frac{\eta_{2}-\eta_{1}}{a}\right)-\arctan \left(\frac{\eta_{2}+\eta_{1}}{a}\right)\right) \delta\left(-\eta_{1}\right)+\right.} \\
&\left.+\left(\frac{a}{a^{2}+\left(\eta_{2}-\eta_{1}\right)^{2}}+\frac{a}{a^{2}+\left(\eta_{2}+\eta_{1}\right)^{2}}\right) H\left(-\eta_{1}\right)\right],
\end{aligned}
$$

and

$$
\begin{aligned}
u_{2}=-\frac{A P c_{R}^{2} a}{4 \pi \varepsilon c_{2}^{2}} & {\left[\left(\arctan \left(\frac{\eta_{2}-\eta_{1}}{a}\right)-\arctan \left(\frac{\eta_{2}+\eta_{1}}{a}\right)\right) \delta\left(-\eta_{1}\right)-\right.} \\
& \left.-\left(\frac{a}{a^{2}+\left(\eta_{2}-\eta_{1}\right)^{2}}-\frac{a}{a^{2}+\left(\eta_{2}+\eta_{1}\right)^{2}}\right) H\left(-\eta_{1}\right)\right] .
\end{aligned}
$$

Although it is not feasible to evaluate the integral given by (3.18), representing the inner solution, we may still employ numerical integration schemes to illustrate the displacement below the surface some of which are given in Figure 6 .

\section{Numerical results}

In the previous sections we have obtained analytical as well as integral representations both for potentials and displacement components. Our main interest, here, is to represent effect of the loading on the half-space as the parameter $a$ varies. Evidently, for smaller values of $a$ the load exhibits a point load-like behaviour causing a singularity in the displacement components. It is expected that the larger values of $a$ will result in smoothened displacement profiles. In the figures to follow the Poisson ratio is taken as $\nu=0.25$ corresponding to a relation between Rayleigh and transverse wave speeds given by $c_{R}=0.9194 c_{2}$. Also the displacements are scaled as

$$
U_{i}\left(\eta_{1}, \eta_{2}, \eta_{3}\right)=\frac{2 \pi}{A P} u_{i}\left(\eta_{1}, \eta_{2}, \eta_{3}\right), \quad i=1,2,3 .
$$

Let us consider, first, the sub-Rayleigh case where $\varepsilon=0.1$ for which the load speed equals $c=0.924 c_{2}$. In Figure 2 the longitudinal surface displacement $U_{1}$ is plotted along $\eta_{1}$ axis, that is, in the direction of the motion for several values of the parameter $a$. It is also interesting to note that a simple analysis reveals that the particular value of $a=1$ is a second order zero of the derivative of $U_{1}$ which accounts for the behaviour of the graph of $U_{1}$ around the origin. Figure 3 represents variation of the vertical displacement $U_{3}$ with respect to $\eta_{2}$ on the surface $\eta_{3}=0$. It is clearly seen, in both of the graphs, that an increase in the value of parameter $a$ diminishes the singularity of the displacements, whereas in the limit $a \rightarrow 0$, the displacement profiles exhibit a singularity under the load encountered in point force problems (cf. [16]).

Figure 4 shows the cross-section of the vertical displacement $U_{3}$ for $\eta_{2}=2$, depending on depth, when $a=0.01$ (Figure 4a) and $a=0.1$ (Figure 4b). It is observed that even for small values of $a$, the singularity at the near-surface vanishes rapidly. 


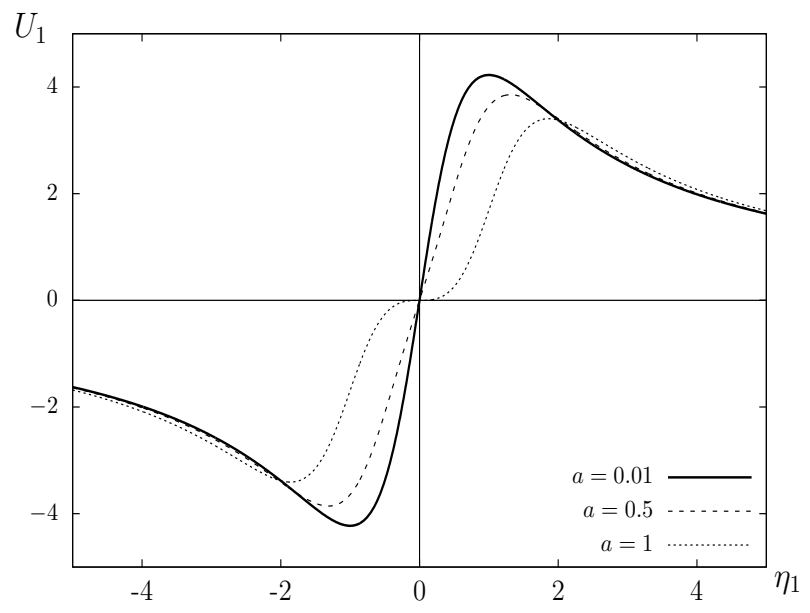

Figure 2. Sub-Rayleigh surface displacement $U_{1}$ versus $\eta_{1}$ for $\eta_{2}=1$ and $a=0.01, a=0.5, a=1$.

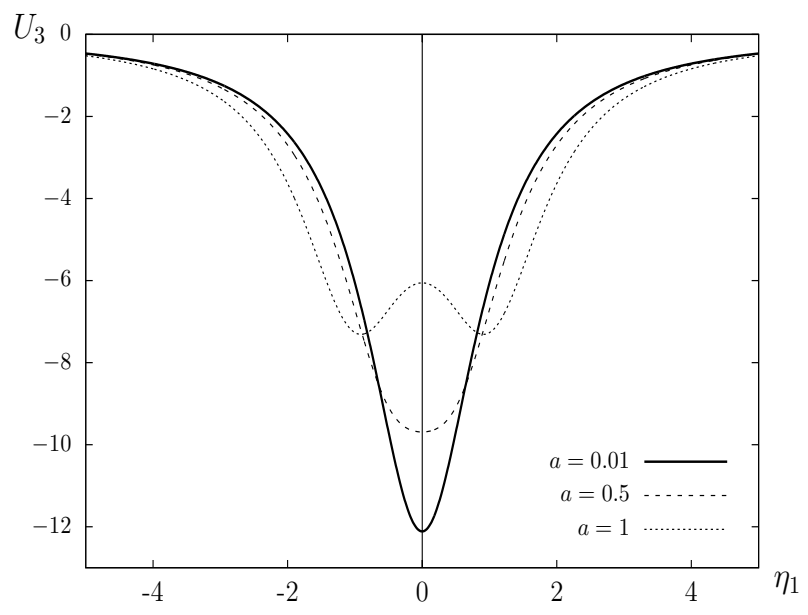

Figure 3. Sub-Rayleigh vertical surface displacement $U_{3}$ versus $\eta_{1}$ for $\eta_{2}=0.1$ and $a=0.01, a=0.5, a=1$.

We now investigate the super-Rayleigh case in which the load speed is taken as $c=$ $0.924 c_{2}$. In Figure 5 the effect of the parameter $a$ on the displacements $U_{1}$ and $U_{3}$ along the surface is illustrated. It is apparent that a larger Gaussian-like profile of the load, i.e., increasing values of $a$ decreases the size of the singularity under the load. Both Figure 5 (a) and 6 signify that the principle in-plane displacement $U_{1}$ is causal on the surface, however as the depth increases it becomes non-causal. This is a result of the fact that only the longitudinal potential $\varphi$ is causal along the direction of the motion and only on the surface in the super-Rayleigh case which is a consequence of the adapted approximate model (see [9] for further details). A cross-section of the surface longitudinal displacement $U_{1}$ for $n_{1}=-1$ is depicted in Figure 7. Evidently, as $a \rightarrow 0$ a singular behaviour arises 


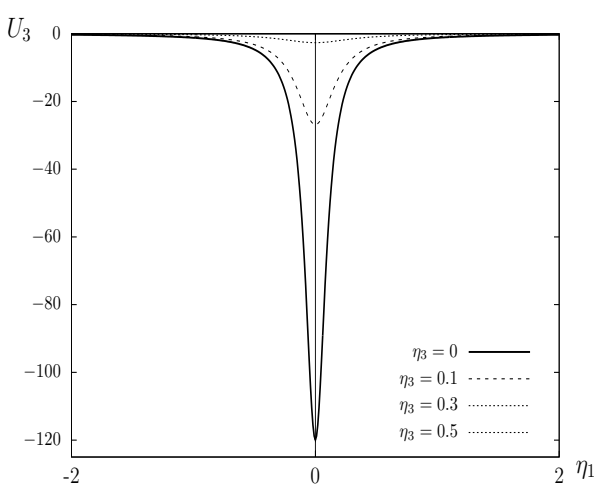

(a)

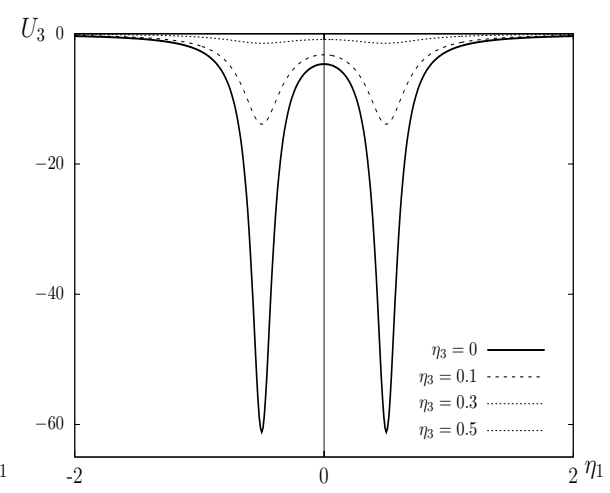

$(b)$

Figure 4. Profiles of the sub-Rayleigh vertical displacement $U_{3}$ versus $\eta_{1}$ for $\eta_{2}=1$ depending on depth; (a) $a=0.01$, (b) $a=0.5$.

under the load exhibiting surface discontinuities at $\eta_{2}= \pm \eta_{1}=-1$, relating to the Mach cone lines behind the load. As mentioned in the foregoing discussion, the causality principle prevents having these lines in front of the load $\left(\eta_{1}>0\right)$. Once again, a larger Gaussian profile of the load smoothens the singularities.

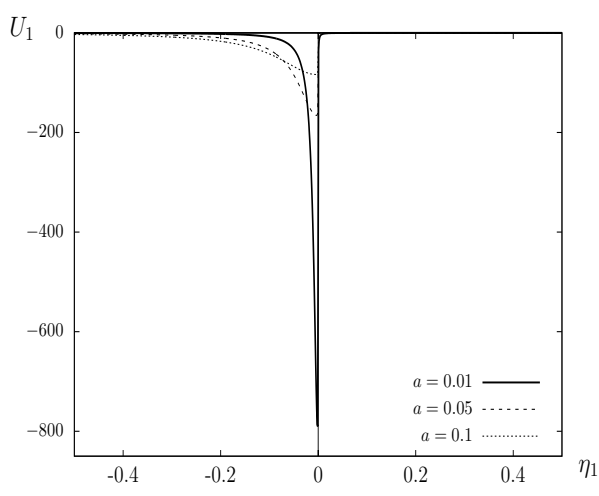

(a)

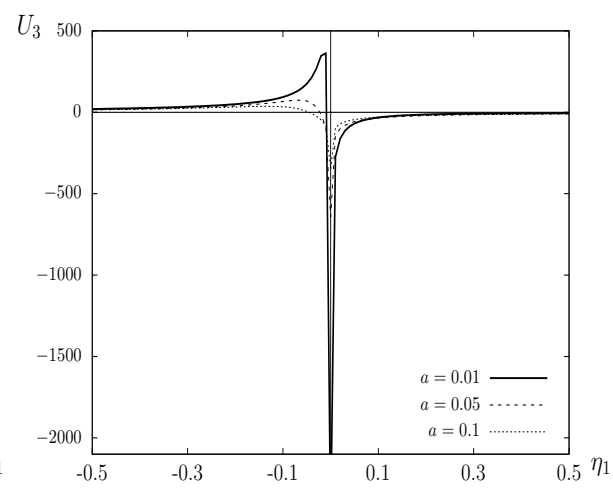

(b)

Figure 5. Profiles of the super-Rayleigh surface displacement components versus $\eta_{1}$ for $\eta_{2}=0$ and $a=0.01, a=0.5, a=1$; (a) horizontal displacement $U_{1}$ (b) vertical displacement $U_{3}$. 


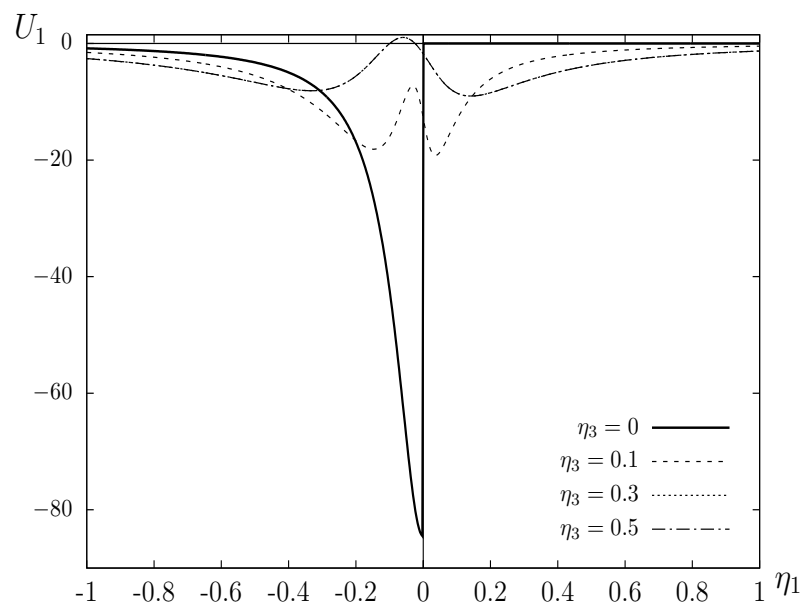

Figure 6. Profiles of the super-Rayleigh in-plane horizontal displacement $U_{1}$ versus $\eta_{1}$ for $\eta_{2}=0$ and $a=0.1$ depending on depth.

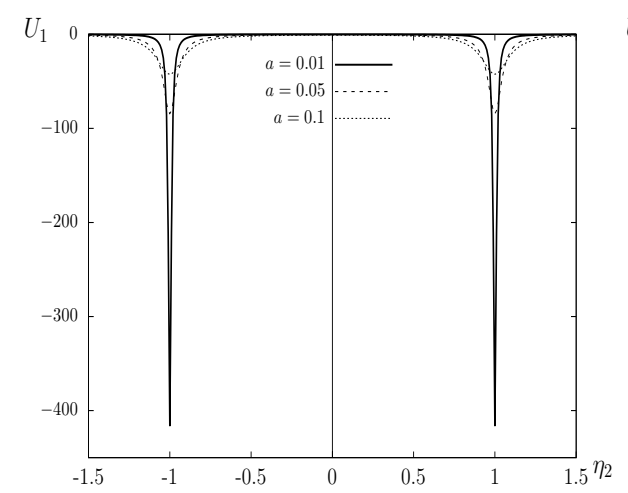

(a)

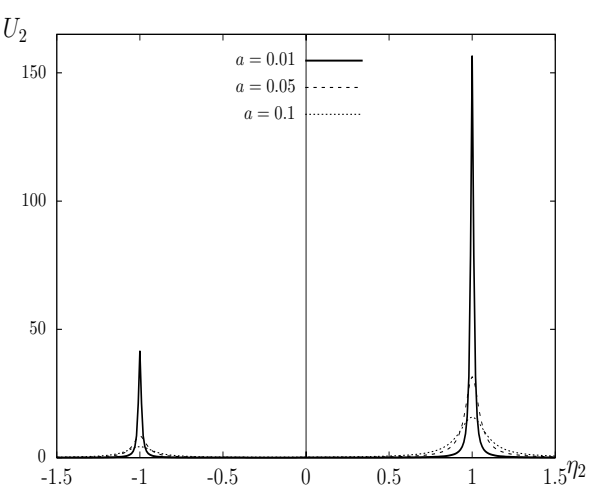

(b)

Figure 7. Profiles of the super-Rayleigh surface in-plane displacement components versus $\eta_{2}$ for $\eta_{1}=-1$ and $a=0.01, a=0.5, a=1$; (a) horizontal displacement $U_{1}$ (b) transverse displacement $U_{2}$.

\section{Conclusions}

This paper focuses on the effect of a distributional load acting on the surface of an elastic half-space. In obtaining the solution of the 3D elliptic problems the hyperbolic-elliptic model given in [15] is employed. This model, taking advantage of the small parameter expressing the proximity of the load speed to the Rayleigh wave speed, concentrates on extracting the effect of the surface waves by enabling the reduction of $3 \mathrm{D}$ problems to $2 \mathrm{D}$ ones. 
The displacements for the sub-Rayleigh case are expressed through elementary functions over the elastic half-space, however, in the super-Rayleigh case, the considered load's distribution along the $x_{2}$ axis causes serious difficulties in the analytical evaluation of integrals for $\varphi$ which prohibits obtaining explicit expressions for the displacements which is in contrast to the case considered in [9]. This necessitates the use of numerical integration to acquire the solutions over the interior. Nevertheless, it is still possible to represent surface displacements through simple analytical expressions (cf. (3.20) and $(3.21))$.

It is known that, in the limit $a \rightarrow 0$ the distributional load coincides with the point force which has been investigated in several publications (see, for instance, [4],[12] and [16]). In the Figures represented for both sub and super-Rayleigh cases in the previous section, it is decidedly seen that the singularities encountered in the point force problems are smoothened or even diminished in the case of a distributed load. It is also worth mentioning that the influence of a distributed load has, somewhat, the same effect of an elastic coating on a half-space (cf. [12])

A number of diverse problems, such as wave propagation in layered media, inhomogeneous elastic beams etc., as well as media where the effects of prestress anisotropy are crucial, may be analysed through a similar approach considered in this work.

\section{Acknowledgements}

The authors are grateful to J.D. Kaplunov for fruitful discussions. B.E. and N.E. are indebted to the Scientific Research Projects of Anadolu University, No: 1408F370, for its financial support.

\section{References}

[1] Cole, J. and Huth, J. Stresses produced in a half plane by moving loads, J. Appl. Mech. 25, 433-436, 1958.

[2] Freund, L. B. Wave motion in an elastic solid due to a nonuniformly moving line load, Quart. Appl. Math. 30, 271-281, 1972.

[3] Fryba, L. Vibration of solids and structures under moving loads (Thomas Telford, London, 1999).

[4] Kaplunov, J., Nolde, E. and Prikazchikov, D. A. A revisit to the moving load problem using an asymptotic model for the Rayleigh wave, Wave Motion 47 (7), 440-451, 2010.

[5] Cao, Y., Xia, H. and Li, Z. A semi-analytical/FEM model for predicting ground vibrations induced by high-speed train through continuous girder bridge, Journal of Mechanical Science and Technology 26 (8), 2485-2496, 2012.

[6] Celebi, E. Three-dimensional modelling of train-track and sub-soil analysis for surface vibrations due to moving loads, Applied Mathematics and Computation 179 (1), 209-230, 2006.

[7] Hackenberg, M. and Müller, G. Modeling a Halfspace with Tunnel using a Coupled Integral Transform Method-Finite Element Method Approach, PAMM 15 (1), 389-390, 2015.

[8] Zhu, X. Q. and Law, S. S. Dynamic load on continuous multi-lane bridge deck from moving vehicles, Journal of Sound and Vibration 251 (4), 697-716, 2002.

[9] Erbaş, B. and Şahin, O. On the causality of the Rayleigh wave, Journal of Mechanics of Material and Structures 11 (4), 449-461, 2016.

[10] Kaplunov, J. and Prikazchikov, D. Explicit models for surface, interfacial and edge waves, in: Dynamic Localization Phenomena in Elasticity, Acoustics and Electromagnetism (Craster, R. V. and Kaplunov, J., eds.) CISM Courses and Notes, 547 (Springer, 2013), 73-114.

[11] Dai, H. H., Kaplunov, J. and Prikazchikov, D. A. A long-wave model for the surface elastic wave in a coated half-space, Proc. R. Soc. A. 466 (2122), 3097-3116, 2010. 
[12] Erbaş, B., Kaplunov, J., Prikazchikov, D. A. and Şahin O. The near-resonant regimes of a moving load in a $3 D$ problem for a coated elastic half space, Math. Mech. Solids DOI: 10.1177/1081286514555451, 2010.

[13] Ege, N., Erbaş, B. and Prikazchikov, D. A. On the 3D Rayleigh wave field on an elastic half-space subject to tangential surface loads, ZAMM-Journal of Applied Mathematics and Mechanics/Zeitschrift für Angewandte Mathematik und Mechanik 95 (12), 1558-1565, 2015.

[14] Achenbach, J. Wave propagation in elastic solids (Elsevier, 2012).

[15] Kaplunov, J., Zakharov, A. and Prikazchikov, D. A. Explicit models for elastic and piezoelastic surface waves, IMA J. Appl. Math. 71 (5), 768-782, 2006.

[16] Kaplunov, J., Prikazchikov, D. A., Erbaş, B. and Şahin, O. On a 3D moving load problem for an elastic half space, Wave Motion 50 (8), 1229-1238, 2013.

[17] Zauderer, E. Partial differential equations of applied mathematics (Vol. 71, John Wiley \& Sons, 2011).

[18] Courant, R. and Hilbert, D. Methods of Mathematical Physics (Vol. 2, John Wiley \& Sons, 1989).

[19] Chadwick, P. Surface and interfacial waves of arbitrary form in isotropic elastic media, J. of Elasticity 6 (1), 73-80, 1976. 